\title{
Quadratic Residues and the Distribution of Primes
}

\section{By Daniel Shanks}

1. Introduction. Chebyshev stated, [1], that there were "many more" primes of the form $4 m-1$ than of the form $4 m+1$ and he indicated, [2], at least three senses in which this assertion was to be understood. If the numbers of primes in these two classes which are $\leqq n$ are designated as $\pi_{-}(n)$ and $\pi_{+}(n)$ respectively, and if

$$
\Delta(n)=\pi_{-}(n)-\pi_{+}(n)
$$

and

$$
t(n)=\frac{\Delta(n)}{\sqrt{n} / \log n},
$$

then Chebyshev stated that for a certain subsequence, $n_{i}$; of the integers,

$$
\operatorname{Lim}_{i \rightarrow \infty} t\left(n_{i}\right)=+1 \text {. }
$$

Since $\sqrt{n} / \log n \rightarrow \infty, \pi_{-}(n)$ could thus be made to exceed $\pi_{+}(n)$ by any amount by an appropriate choice of $n$.

This theorem (which was one of the above-mentioned three senses) was proven by Phragmén, [3], and later by Landau, [4], [5]. The other two "senses" have never been proven, [5, p. 647]. Since the theorem, (3), is entirely noncommittal as to the behaviour of $\Delta(n)$, or of $t(n)$, for values of $n$ other than those of the subsequence $n_{i}$, it is however not a very convincing argument in favor of Chebyshev's "many more" primes assertion. Littlewood, in fact, showed, [6], that there is a constant $K$ such that for two other sequences $n_{j}$ and $n_{k}$ we could have

$$
t\left(n_{j}\right)>K \log \log \log n_{j}
$$

and

$$
t\left(n_{k}\right)<-K \log \log \log n_{k} .
$$

Since $\log \log \log n \rightarrow \infty$ (with great dignity) these inequalities imply that the right side of (3) may be changed from +1 to any real number, positive or negative, for some sequence, $n_{l}$. Because of this Cramér wrote, [7]:

"Chebyshev's general assertion-'there are many more primes of the form $4 n+3$ than of the form $4 n+1$ '-can therefore be true only in a rather limited region."

The purpose of this paper, [8], is to determine the sense in which Chebyshev's general assertion is (nonetheless) probably true, and to identify the nature of the cause which tends to diminish the class of $4 n+1$ primes relative to the $4 n-1$ primes. The problem is generalized to primes, and also to composites, in any arith- 
metic progression. Finally, there are some comments concerning the Littlewood phenomena, $\pi(n)>\operatorname{Li}(n)$.

2. The Function $\Delta(n)$. Tables of $\pi_{-}(n)$ and of $\pi_{+}(n)$, [9], [10], [11], [12], published prior to the short note of John Leech, [13], were not of sufficient completeness (or, in one case, of sufficient accuracy) to correctly identify Cramerr's "rather limited region." Leech computed (on EDSAC) $\Delta(n)$ for $n \leqq 3,000,000$ and determined that 26,861 was the smallest* $n$ for which $\pi_{+}(n)>\pi_{-}(n)$ :

$$
\Delta(26,861)=-1 \text {. }
$$

The author, unaware of Leech's note, carried out a similar computation (on an IBM 704). The following description of $\Delta(n)$ in this range, 1 to $3 \cdot 10^{6}$, agrees (where it overlaps) with the shorter account of Leech. In Table 1 we divide this range into six regions and in each region give the maximum and minimum values of $\Delta(n)$, the number of intervals during which $\Delta(n)$ remains zero, positive, or negative, and the total number of $n$ 's for which $\Delta(n)$ is zero, positive, or negative. The final figures show that $\Delta(n)>0$ for $99.84 \%$ of the $n \leqq 3 \cdot 10^{6}$. Table 2 identifies some extrema (and some zeros) of $\Delta(n)$.

This detailed description makes it highly plausible that the predominantly positive character of $\Delta(n)$ in this range of $n$ is not merely a passing fancy of the integers (as is almost implied by Cramér's remark) but a permanent phenomenon

TABLE 1

Description of $\Delta(n)$ for $1 \leqq n \leqq 3,000,000$

\begin{tabular}{rr|r|r|r|r|r|r|r|r}
\hline \multicolumn{2}{c|}{ Regions of $n$} & Max & Min & $\begin{array}{r}0 \text { In- } \\
\text { tervals }\end{array}$ & + Int. & - Int. & 0 n's & $+n$ 's & $-n$ \\
\hline $1-$ & 462 & +6 & 0 & 5 & 4 & 0 & 10 & 452 & 0 \\
$463-$ & 26,832 & +31 & +1 & 0 & 1 & 0 & 0 & 26,370 & 0 \\
$26,833-\quad 26,926$ & +1 & -1 & 6 & 4 & 1 & 60 & 32 & 2 \\
$26,927-\quad 616,768$ & +105 & +1 & 0 & 1 & 0 & 0 & 589,842 & 0 \\
$616,769-\quad 633,882$ & +12 & -8 & 101 & 48 & 52 & 1,282 & 12,428 & 3,404 \\
$633,883-3,000000+$ & +256 & +1 & 0 & 1 & 0 & 0 & $2,366,118$ & 0 \\
\hline
\end{tabular}

TABLE 2

Some Special Values

$$
\begin{aligned}
& \Delta(227)=+6 \\
\Delta(461)= & \Delta(462)=0 \\
& \Delta(17,971)=+31 \\
\Delta(26,861)= & \Delta(26,862)=-1 \\
& \Delta(359,327)=+105 \\
& \Delta(623,681)=-8 \\
\Delta(627,859)=\cdots= & \Delta(627,900)=0 \\
& \Delta(2,951,071)=+256
\end{aligned}
$$

* This first axis crossing of $\Delta(n)$ was discovered independently by J. W. Wrench, Jr. 
for which a sufficient number-theoretic cause should be assigned and of which a more precise formulation is desirable.

3. The Function $\tau(n)$. We therefore return to $t(n)$ and seek a better insight into this function. We do not compute $t(n)$ itself, but instead, the somewhat simpler:

$$
\tau(n)=\Delta(n) \cdot \sqrt{n} / \pi(n)
$$

where $\pi(n)$ is the total number of primes $\leqq n$. From the prime number theorem, $\pi(n) \sim n / \log n$, we have

$$
\tau(n) \sim t(n)
$$

For example, for $n=2,000,000$ we have

$$
\begin{array}{rlrl}
\pi_{-}(n) & =74,516 ; & \pi_{+}(n)=74,416 ; & \pi(n)=148,933 ; \\
\Delta(n)=100 ; & \tau(n)=0.9496 ; & \text { and } \quad t(n)=1.0259 .
\end{array}
$$

The function $\tau(1000 k)$ was computed for the 2000 values $k=1,2, \cdots, 2000$. The minimum, the mean, and the maximum of these 2000 values are

$$
\begin{aligned}
\tau(629,000) & =-0.0464, \\
\frac{1}{2000} \sum_{1}^{2000} \tau(1000 k) & =1.0613, \\
\tau(127,000) & =2.0961,
\end{aligned}
$$

respectively. Only slightly greater extremes would have been obtained had we computed $\tau(n)$ for all the $2 \cdot 10^{6} n$ in this range. The distribution of these 2000 values of $\tau$ between the extremes was determined by counting the $k$ 's for which

$$
\frac{m}{16} \leqq \tau(1000 k)<\frac{m+1}{16} \quad(m=-1,0,1, \cdots, 33) .
$$

These counts, $\nu(m)$, are tabulated in Table 3 and plotted in a bar graph in Fig. 1.

The following comments are now in order:

a.) The distribution is roughly normal with a mean of (nearly) +1 .

b.) The rare cases of $\Delta(n)<0$ (i.e., $\tau(n)<0$ ) are now to be thought of as no more unusual than the equally rare other extreme: $\Delta(n)>2 \pi(n) / \sqrt{n}$ (i.e., $\tau(n)>2$ ).

c.) The implication of Littlewood's inequalities, (4) and (5), is that the distribution function has tails of infinite extent. Of course, occurrences of $\tau$ far out in a tail will be very rare.

TABLE 3

\begin{tabular}{l|r|r|r|r|r|r|r|r|r|r|r|r}
\hline $\begin{array}{l}m \\
\nu(m)\end{array}$ & -1 & 0 & 1 & 2 & 3 & 4 & 5 & 6 & 7 & 8 & 9 & 10 \\
\hline$m$ & 11 & 12 & 13 & 14 & 15 & 16 & 17 & 18 & 19 & 20 & 21 & 22 \\
$\nu(m)$ & 76 & 114 & 139 & 156 & 139 & 151 & 152 & 136 & 129 & 109 & 107 & 77 \\
\hline$m$ & 23 & 24 & 25 & 26 & 27 & 28 & 29 & 30 & 31 & 32 & 33 & \\
$\nu(m)$ & 52 & 51 & 55 & 29 & 21 & 19 & 17 & 12 & 5 & 0 & 2 & \\
\hline
\end{tabular}




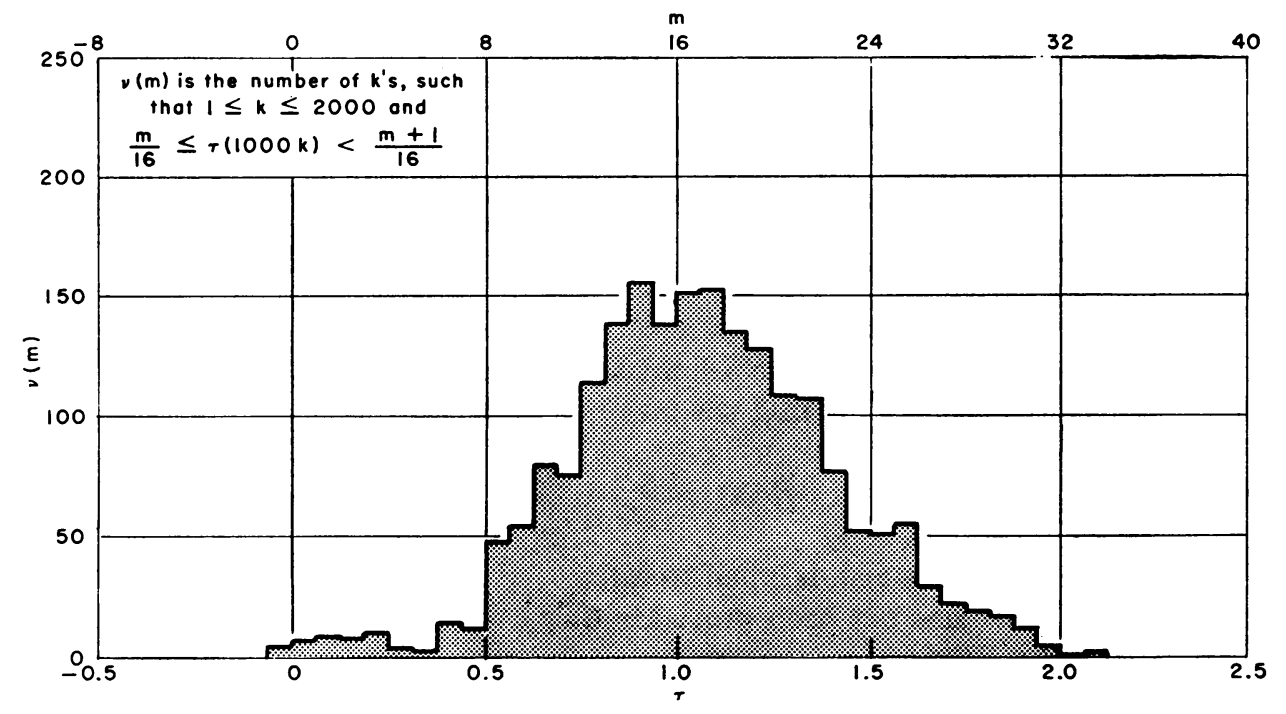

Fig. 1-The distribution of 2000 values of $\tau(1000 k)$

d.) Chebyshev's function $\sqrt{n} / \log n$ (or its equivalent $\pi(n) / \sqrt{n}$ ) is seen to be an appropriate normalizing factor-it reduces the function $\Delta(n)$ to a function of $n$ with relatively small variation. For example, throughout most of the second million, $(1,015,000$ to $2,000,000), \tau(1000 k)$ has the following very modest behaviour:

$$
\tau(1,811,000)=0.426 \leqq \tau(1000 k) \leqq 1.610=\tau(1,521,000) .
$$

e.) The function

$$
\tau_{s}=\frac{1}{50 s} \sum_{k=1}^{k=50 s} \tau(1000 k) \quad(s=1,2, \cdots, 40)
$$

provides a running history of the mean value of $\tau$, and is found to change very little:

$$
\tau_{15}=1.0222 \leqq \tau_{s} \leqq 1.1799=\tau_{3} \quad(s=1,2, \cdots, 40) .
$$

4. A Conjecture. The above discussion suggests that the proper formulation of Chebyshev's "many more" primes assertion is the following

Conjecture. The mean value of $\tau(n)$,

$$
\frac{1}{N-1} \sum_{2}^{N} \tau(n)
$$

has a limit as $N \rightarrow \infty$ and this limit equals +1 ;

$$
\operatorname{Lim}_{N \rightarrow \infty} \frac{1}{N-1} \sum_{2}^{N} \tau(n)=+1 \text {. }
$$

The conjectured limit, +1 , may seem a little rash in view of the limited data, (12). However, there is other evidence. The remark made after (5) may suggest 
that the +1 right side of $(3)$ is of no significance since it may be replaced by any real number. Nonetheless, from Landau's proof, [5], of (3) it is readily seen that +1 has a very special role. For every $a$ the Dirichlet series

$$
F_{a}(s)=\sum_{n=2}^{\infty} \frac{\Delta(n)-a \sqrt{n} / \log n}{n^{s+1}}
$$

defines a function which is regular in the half plane $R(s)>1$ and for the real values $\frac{1}{2}<s \leqq 1$, but is singular for $s=\frac{1}{2}$. However, only for $a=1$, that is, only for $F_{1}(s)$, does the function have a limit as $s \rightarrow \frac{1}{2}+$. Using this fact, and $\tau(n) \sim$ $t(n)$, what is to be proved is that the mean value of the $a_{n}$ in

$$
F_{1}(s)=\sum_{n=2}^{\infty} \frac{a_{n}}{n^{s+t} \log n}
$$

has a limit as $n \rightarrow \infty$ which is equal to zero.

The conjecture has not been proved, either as its stands, or in one of the two following weaker forms:

Conjecture. If no zero of

$$
L(s)=1-\frac{1}{3^{s}}+\frac{1}{5^{s}}-\frac{1}{7^{s}}+\frac{1}{9^{s}}-\cdots
$$

has a real part $>\frac{1}{2}$, then (13) is true.

Conjecture. If

$$
\operatorname{Lim}_{N \rightarrow \infty} \frac{1}{N-1} \sum_{2}^{N} \tau(n)
$$

exists, it equals +1

However, even the original conjecture seems sufficiently likely to be true to merit recording. The closest thing to it, in the literature known to the author, is Theorem 2.351 of Hardy and Littlewood, [6, p. 151]. This, together with their Theorem 2.34 and a third result due to Landau, [7], may be combined and entirely rewritten to read:

Theorem. If no zero of $L(s)$ has a real part $>\frac{1}{2}$, then there is a positive $K$ such that

$$
\frac{1}{N} \sum_{1}^{N} \Delta(n)>K \frac{\sqrt{N}}{\log N}
$$

for all sufficiently large $N$, and conversely.

From this theorem, Abel's lemma on partial summation, [14], and (8), we can easily prove the following

Corollary. If no zero of $L(s)$ has a real part $>\frac{1}{2}$, then there is a positive $K$ such that

$$
\frac{1}{N-1} \sum_{2}^{N} \tau(n)>K
$$

for all sufficiently large $N$.

We will present further evidence for the conjecture, in section 9 below, after we have determined the number-theoretic cause of the $4 m+1$ prime deficiency. 
5. Localization of the Deficiency. If the classes $4 m+1$ and $4 m-1$ are split, modulo 8 , we have

$$
\overbrace{8 m+1}^{4 m+1} \frac{4 m-1}{8 m+5} \quad \overbrace{8 m+3}^{4 m+7}
$$

and we now inquire whether the deficiency exhibited by the $4 m+1$ primes is shared equally by the $8 m+1$ and the $8 m+5$ primes. Landau's generalization of (3) for any modulus, [5, p. 704-711], and other simpler considerations given below, suggest, on the contrary, that the deficiency is confined entirely to $8 m+1$.

Let $\pi_{a, b}(n)$ be the number of primes of the form $a m+b$ which are $\leqq n$. A table of $\pi_{8, b}(1000 k)$ was computed for $b=1,3,5$, and 7 and $k=1,2, \cdots, 1000$. Examination of this table shows that while the numbers of primes of the forms $8 m+3,8 m+5$, and $8 m+7$ are continually jockeying for first place, the number of $8 m+1$ primes is always in last place. Let $f_{b}(n)$ be the fraction of the tabular values, with argument $\leqq n$, for which $a m+b$ is in first place. (Two-way or threeway ties for first place are pro-rated $\frac{1}{2}$ or $\frac{1}{3}$ of a point respectively.) Similarly let $l_{b}(n)$ be the fraction of the tabular values for which $a m+b$ is in last place. In the present case, since $8 m+1$ is in last place throughout the entire range of the table, we have for every $k=1,2, \cdots, 1000$ :

$$
f_{1}(1000 k)=l_{3}(1000 k)=l_{5}(1000 k)=l_{7}(1000 k)=0 .
$$

A brief summary of $\pi_{8, b}$ and the four other $f_{b}$ and $l_{b}$ functions is shown in Table 4 . No significant deviations from equality, [15], are to be noted among $8 m+3$, $8 m+5$, and $8 m+7$, - the slightly low $f_{5}(1,000,000)$ is compensated by the slightly high $f_{5}(750,000)$. We conclude that the deficiency of the $4 m+1$ primes resides solely in the $8 m+1$ primes. On those rare occasions (regions 3 and 5 of Table 1) when $\pi_{+}(n)>\pi_{-}(n)$, we invariably find that $8 m+5$ is in first place with a sufficient lead over $8 m+3$ and $8 m+7$ to overcompensate for the leads which these latter classes hold over $8 m+1$. It is not known whether $8 m+1$ itself can ever take the lead but in view of what is written below about $24 m+1$, this is probable - for sufficiently large $n$.

TABLE 4

\begin{tabular}{|c|c|c|c|c|c|c|c|c|}
\hline$n$ & $\pi 8,1$ & $\pi 8,3$ & $\pi 8,6$ & $\pi 8.7$ & $l_{1}$ & $f_{3}$ & $f_{\mathbf{b}}$ & $f_{7}$ \\
\hline 250,000 & 5473 & 5525 & 5523 & 5522 & 1.000 & 0.446 & 0.322 & 0.232 \\
\hline 500,000 & 10334 & 10418 & 10397 & 10388 & 1.000 & 0.337 & 0.344 & 0.319 \\
\hline 750,000 & 14998 & 15087 & 15078 & 15074 & 1.000 & 0.380 & 0.382 & 0.237 \\
\hline $1,000,000$ & 19552 & 19653 & 19623 & 19669 & 1.000 & 0.401 & 0.287 & 0.312 \\
\hline
\end{tabular}

Distribution of the Primes Modulo 8

6. Distinctions between Modulo 12 and Modulo 10. Alternatively, we could have split $4 m+1$ and $4 m-1$ modulo 12 :

$$
\overbrace{12 m+1}^{4 m+1} \overbrace{12 m+7}^{4 m-1} \frac{4 m+12 m+11}{12 m+5}
$$


TABLE 5

Distribution of the Primes Modulo 12

\begin{tabular}{|c|c|c|c|c|c|c|c|c|}
\hline$n$ & $\pi 12,1$ & $\pi 12,5$ & $\pi 12,7$ & $\pi 12,11$ & $l_{1}$ & $f_{6}$ & $f_{7}$ & $f_{11}$ \\
\hline 252,000 & '5504 & 5567 & 5564 & 5566 & 1.000 & 0.352 & 0.420 & 0.228 \\
\hline 504,000 & 10404 & 10476 & 10480 & 10472 & 1.000 & 0.231 & 0.274 & 0.495 \\
\hline 756,000 & 15100 & 15196 & 15204 & 15186 & 1.000 & 0.396 & 0.258 & 0.346 \\
\hline $1,008,000$ & 19715 & 19771 & 19812 & 19797 & 1.000 & 0.338 & 0.402 & 0.260 \\
\hline
\end{tabular}

TABLE 6

Distribution of the Primes Modulo 10

\begin{tabular}{|c|c|c|c|c|c|c|c|c|}
\hline$n$ & $\pi 10,1$ & $\pi 10,8$ & $\pi 10,7$ & $\pi 10,9$ & $l_{1}$ & $f_{3}$ & $f_{7}$ & $l_{9}$ \\
\hline 250,000 & 5495 & 5520 & 5517 & 5510 & 0.492 & 0.556 & 0.444 & 0.508 \\
\hline 500,000 & 10386 & 10382 & 10403 & 10365 & 0.555 & 0.439 & 0.561 & 0.445 \\
\hline 750,000 & 15027 & 15084 & 15073 & 15052 & 0.533 & 0.445 & 0.555 & 0.467 \\
\hline $1,000,000$ & 19617 & 19665 & 19621 & 19593 & 0.502 & 0.582 & 0.418 & 0.498 \\
\hline
\end{tabular}

A table of $\pi_{12, b}(1008 k)$ was computed for $b=1,5,7$, and 11 and $k=1,2, \cdots, 1000$. (An interval of 1008 was chosen, since its divisibility by 12 simplified the computer program and eliminated even slight inequalities.) The results are similar: the deficiency of $4 m+1$ resides solely in $12 m+1$, and when $\Delta(n)<0$ we now find $12 m+5$ in the lead. Again,

$$
f_{1}=l_{5}=l_{7}=l_{11}=0
$$

throughout the range computed. Again, the three stronger classes are all equally strong-see Table 5.

In contrast now consider the four classes of primes modulo $10,10 m+1,+3$, +7 , and +9 . This time we find

$$
f_{1}(1000 k)=l_{3}(1000 k)=l_{7}(1000 k)=f_{9}(1000 k)=0
$$

for $k=1,2, \cdots, 1000$ so that for these arguments neither $10 m+1$ nor $10 m+9$ is ever in first place. ${ }^{*}$ We find that $10 \mathrm{~m} \pm 3$ take turns in first place while $10 \mathrm{~m} \pm 1$ take turns in last place. From Table 6 we conclude that this time we have two strong classes (which are equally strong), $10 \mathrm{~m} \pm 3$, and two weak classes (which are equally weak), $10 \mathrm{~m} \pm 1$.

These striking distinctions between modulo 8 and 12 on the one hand and modulo 10 on the other are easily explained in terms of the corresponding modulo multiplication groups, and this explanation provides us with a simple, numbertheoretic, sufficient cause.

7. Group Multiplication and Fluctuations. Consider the multiplication table modulo 8 of the four residue classes $1,3,5$, and 7

* At some intermediate arguments, e.g., $n=135852,10 m+1$ is tied for first place and similarly, for $n=969240,10 m+7$ is tied for last place. 


\begin{tabular}{|c|c|c|c|}
\hline 1 & 3 & 5 & 7 \\
\hline 3 & 1 & 7 & 5 \\
\hline 5 & 7 & 1 & 3 \\
\hline 7 & 5 & 3 & 1 \\
\hline
\end{tabular}

and assume that in an interval centered around $n$ there are fewer primes of the form $8 m+1$ than of the other three types. Consider the excess primes of the other three classes. Their products with themselves and with each other are composites (of order $n^{2}$ ) whose residue classes are contained in the lower right $3 \times 3$ box. Now note that these products are distributed into the four residue classes in proportions $3: 2: 2: 2$ - there is an extra product congruent to 1 . Assume on the contrary an excess number of primes of the form $8 m+1$. The composites from these primes lie in the upper left $1 \times 1$ box. Again we find an excess of composites congruent to 1 . Similarly, too few or too many primes congruent to 3,5 , or 7 will also lead to an excess number of composites congruent to 1 . Briefly, any fluctuation from equality in the distribution of the primes into the four residue classes will create an excess number of composites and therefore a diminished number of primes congruent to 1 modulo 8 . This idea, that the phenomenon in question is essentially a fluctuation phenomenon (with analogies to the fluctuation phenomena of physics), arose during a discussion of this problem between the author and T. S. Walton. The idea is thus partly due to him.

Since the multiplication group modulo 12 is isomorphic to that modulo 8 , the behaviour found modulo 12 is similar. But for 10 we do not have the "vier" group but the cyclic group

\begin{tabular}{|c|c|c|c|}
\hline 1 & 3 & 7 & 9 \\
\hline 3 & 9 & 1 & 7 \\
\hline 7 & 1 & 9 & 3 \\
\hline 9 & 7 & 3 & 1 \\
\hline
\end{tabular}

and this time we find that fluctuations in $10 m+3$ or $10 m+7$ diminish the $10 m+9$ primes while fluctuations in $10 m+1$ or $10 m+9$ diminish the $10 m+1$ primes. 
8. Quadratic Residues and the Distribution of Primes. In general, it is readily seen, those residue classes which occur on the principal diagonal are the ones whose numbers of primes are diminished. These are the quadratic residues. We expect then, for any modulus, that the prime race will separate (in the mean) into two racesthat between the non-residues, up front, and that between the residues, in the rear. In particular, since +1 is the only quadratic residue of 24 , the deficiency of $4 m+1$, which was found to reside solely in $8 m+1$ or $12 m+1$, can be further localized to their intersection, $24 m+1$.

For most larger moduli, unlike the unusual 24 , there will be many quadratic residues and the deficiency, $\sqrt{n} / \log n$, will be shared among all of them. Thus the separation between the residues and the non-residues will not be as sharp, and extensive interplay is to be expected.

9. Propagation of the Deficiency and Higher Order Effects. The quadratic fluctuation effect just discussed is not merely strong enough to maintain the mean deficiency in the $4 m+1$ primes, but, as we shall see presently, as $n$ increases it becomes too strong and we must examine the compensating cubic and higher order effects in order to obtain an accurate picture. We confine ourselves to the modulus 4 , although the generalization offers little difficulty. We have seen, (9), that the mean value of $\tau$ up to $n=2 \cdot 10^{6}$ is nearly one and we wish to show how a mean value equal to one can propagate itself to larger values of $n$. Our computation, however, is only approximate, and while the result gives further evidence for the truth of (13), it is not a proof of that conjecture.

Let $a$ be a positive integer and let $\pi_{+}{ }^{(a)}(n)$ be the number of positive integers of the form $4 m+1$ which are $\leqq n$ and which are the products of $a$ (not necessarily distinct) primes. Our previous $\pi_{+}(n)$ is now $\pi_{+}^{(1)}(n)$. With a similar definition for $\pi_{-}^{(a)}(n)$, let

$$
\begin{aligned}
\Delta_{!}^{(a)}(n) & =\pi_{-}^{(a)}(n)-\pi_{+}^{(a)}(n), \\
\Sigma^{(a)}(n) & =\pi_{-}^{(a)}(n)+\pi_{+}^{(a)}(n),
\end{aligned}
$$

and

$$
\tau^{(a)}(n)=\frac{\Delta^{(a)}(n)}{\Sigma^{(a)}(n)} \sqrt{n}
$$

Note the slight difference between $\tau$ and $\tau^{(1)}$ - the new denominator, $\Sigma^{(1)}(n)=$ $\pi(n)-1$, omits the count of the prime 2 .

We wish to compute the mean value of $\tau^{(1)}(n)$ and we assume that the mean values of $\tau^{(1)}(x)$ for $x=n^{1 / a}(a=2,3,4, \cdots)$ are all equal to 1 . It follows that of all the odd primes of order $n^{1 / a}$, a fraction equal to $\frac{1}{2}\left(1+n^{-1 / 2 a}\right)$ is of the form $4 m-1$ and a fraction equal to $\frac{1}{2}\left(1-n^{-1 / 2 a}\right)$ is of the form $4 m+1$. The composites of order $n$, which are the products of $a$ primes, have as prime factors primes whose (geometric) mean order is $n^{1 / a}$. With reference to the multiplication table $(\bmod 4)$ :

$$
\begin{array}{|l|l|}
\hline+1 & -1 \\
\hline-1 & +1 \\
\hline
\end{array}
$$


we now find, by induction on $a$, that among all possible $a$-fold products of these primes, a fraction equal to $\frac{1}{2}\left(1+(-1)^{a+1} n^{-\frac{1}{2}}\right)$ is of the form $4 m-1$ and a fraction equal to $\frac{1}{2}\left(1-(-1)^{a+1} n^{-\frac{1}{2}}\right)$ is of the form $4 m+1$. In other words, on the average, we will have, for $a=2,3, \cdots$,

$$
\bar{\tau}^{(a)}(n)=(-1)^{a+1}
$$

Thus we find a simple and interesting generalization of the Chebyshev phenomenon. There will be, in the mean, an excess of $4 m-1$ composites of odd degree $a$, and an excess of $4 m+1$ composites of even degree. Further, the mean fractional excess will be $n^{-\frac{1}{2}}$, independent of the degree $a$.

Now, for any $n$,

$$
0=\Delta^{(1)}(n)+\Delta^{(2)}(n)+\Delta^{(3)}(n)+\cdots
$$

with an error of 0 or 1 , and therefore, with the same error,

$$
\Delta^{(1)}(n)=-\Delta^{(2)}(n)-\Delta^{(3)}(n)-\cdots .
$$

As long as $n$ is not too large, say 1000 , most of the composites are of second degree, and we have $\Delta^{(1)}(n) \approx-\Delta^{(2)}(n)$. This, essentially, was our picture in sec. 7 above, But as $n$ increases, the other even degree composites would tend to increase the deficiency while the odd degree composites tend to diminish it. To determine the balance we use the known fact, [5, p. 627], that in any arithmetic progression the number of integers with $a$ odd is asymptotically equal to those with $a$ even. Specifically, for the progression $2 m+1$, we have

$$
(1+\epsilon) \Sigma^{(1)}(n)=\Sigma^{(2)}(n)-\Sigma^{(3)}(n)+\Sigma^{(4)}(n)-\cdots,
$$

where $\epsilon \rightarrow 0$ as $n \rightarrow \infty$. Therefore from (20), (21), and (22) we have, in the mean,

Thus

$$
\Delta^{(1)}(n)=(1+\epsilon) \Sigma^{(1)}(n) / \sqrt{n} \text {. }
$$

and a mean value of one propagates itself.

There are two remarks of interest concerning equations (21) and (22).

1.) The sequences $\chi(n)$ and $\lambda(n)$ are defined as follows:

$$
\lambda(n)=(-1)^{a}
$$

where $a$ is the number of prime factors of $n$, and $\chi(n)=0,1,0$, and -1 for numbers of the form $4 m, 4 m+1,4 m+2$, and $4 m+3$ respectively. Let

$$
b_{n}=\lambda(n) \chi(n) \text {. }
$$

A generalized Chebyshev assertion now reads: there are "many more" integers $m$ for which $b_{m}=+1$ than for which $b_{m}=-1$ and the mean excess is of order $\frac{1}{2} \sqrt{n}$. Since it is known, [5, p. 674], that

$$
f(s)=\sum_{n=1}^{\infty} b_{n} n^{-s}=\left(1-2^{-2 s}\right) \zeta(2 s) / L(s),
$$

it is clear that a function-theoretic formulation of this assertion is concerned with $f(s)$ and with the zeros of $L(s)$. 
2.) Let $n$ be sufficiently large such that the number of primes is small compared with the number of composites. Then the terms of like sign in the right side of (22) could be combined so that the right side would be the difference between two nearly equal numbers. We therefore have an analogue of a difficulty in numerical analysis-subtractive loss of significance. In fact, the analogy is very good, since it is known, [15, p. 342], that the normal order of the number of prime divisors of $n$ is $\log \log n$ and that the number of composites of degree $a$ is given asymptotically by

$$
\pi^{(a)}(n) \sim n(\log \log n)^{a-1} /(a-1) ! \log n .
$$

This means that the numbers of composites of degree $a$ have an approximate Poisson distribution around a mean of $\log \log n$ and that the equation (22) is comparable to the following equation, which is numerically sensitive when $n$ is large:

$$
1-e^{-\log \log n}=\log \log n-\frac{1}{2}(\log \log n)^{2}+\cdots \text {. }
$$

We should therefore expect disturbances in the balance, (23), and more or less random oscillations around this mean. The oscillations should grow as $\log \log n$ increases and may be expected to show strength when the condition $\Sigma^{(1)} \ll$ $\Sigma^{(2)} \approx \Sigma^{(3)}$ is met. This should occur for $\log \log n=2.5+$ or $\log \log \log n \approx 1$.

With these remarks we are led to our final topic.

10. The Littlewood Phenomena. Analogous to Chebyshev's assertion is the erroneous inequality:

$$
\pi(n)<\operatorname{Li}(n)
$$

which was thought to be correct both by Gauss and by Riemann, [7, p. 791, 795]. Again we have the Littlewood counterexample

$$
\pi(n)-L i(n)>K \frac{\sqrt{n}}{\log n} \log \log \log n .
$$

And again we obtain, this time from Riemann's prime formula, [7, p. 795],

$$
\operatorname{Li}(n)=\pi(n)+\frac{1}{2} \pi(\sqrt{n})+\cdots,
$$

and from $\pi(\sqrt{n}) \sim 2 \sqrt{n} / \log n$, the suggestion to define

$$
r(n)=\frac{\operatorname{Li}(n)-\pi(n)}{\sqrt{n} / \log n}
$$

Or similarly let us define

$$
\rho(n)=\frac{L i(n)-\pi(n)}{\pi(n)} \sqrt{n} .
$$

Analogy with (13) now suggests the possibility that

$$
\operatorname{Lim}_{N \rightarrow \infty} \frac{1}{N-1} \sum_{2}^{N} \rho(n)=+1
$$

is the proper formulation of the erroneous (24). 
If, indeed, the differences $L i(n)-\pi(n)$ and $\pi_{-}(n)-\pi_{+}(n)$ are usually of the same sign and order of magnitude we should find that

$$
\pi^{*}(n)=\pi(n)+\pi_{-}(n)-\pi_{+}(n)=2 \pi_{-}(n)+1
$$

agrees with $\operatorname{Li}(n)$ much better than $\pi(n)$ does. This is, in fact, the case as is seen in Table 7, [16]. We therefore regard (29) as plausible. It should be investigated.

A final remark concerning the Littlewood phenomena may be of value. It is sometimes said or implied, [17], that (24) is true "im Bereich der Tabellen", where the tables in question include such entries as $n=10^{8}$ and $n=10^{9}$. But $\pi(n)-L i(n)$ has not been evaluated for all intermediate values of $n$ (say for $10^{8}<n<10^{9}$ ) and consequently, it is not certain that (24) holds there. In the analogous case of $\Delta(n)$ discussed above, had we computed $\Delta(10,000 k)$ up to $k=300$ we would have always found $\Delta>0$ since all the axis crossings of $\Delta(n)$ up to $n=3.10^{6}$ occur at intermediate values of $n$. Further, since the first crossing of $\pi(n)-\operatorname{Li}(n)$ may, like Leech's 26861 above, be of very short duration, it could well be missed unless the computing interval in $n$ were rather small. The author knows of no compelling

TABLE 7

\begin{tabular}{|c|c|c|c|c|}
\hline$n$ & $\pi(n)$ & $\pi^{*}(n)$ & $\pi(n) / \operatorname{Li}(n)$ & $\pi^{*}(n) / \operatorname{Li}(n)$ \\
\hline $10^{3}$ & 168 & 175 & 0.9459 & 0.9853 \\
\hline $2 \cdot 10^{3}$ & 303 & 311 & 0.9625 & 0.9879 \\
\hline $3 \cdot 10^{3}$ & 430 & 437 & 0.9712 & 0.9870 \\
\hline $4 \cdot 10^{3}$ & 550 & 561 & 0.9728 & 0.9923 \\
\hline $5 \cdot 10^{3}$ & 669 & 679 & 0.9777 & 0.9923 \\
\hline $6 \cdot 10^{3}$ & 783 & 799 & 0.9782 & 0.9982 \\
\hline $7 \cdot 10^{3}$ & 900 & 915 & 0.9843 & 1.0007 \\
\hline $8 \cdot 10^{3}$ & 1007 & 1015 & 0.9811 & 0.9889 \\
\hline $9 \cdot 10^{3}$ & 1117 & 1125 & 0.9825 & 0.9895 \\
\hline $10^{4}$ & 1229 & 1239 & 0.9862 & 0.9943 \\
\hline $2 \cdot 10^{4}$ & 2262 & 2273 & 0.9884 & 0.9932 \\
\hline $3 \cdot 10^{4}$ & 3245 & 3267 & 0.9903 & 0.9970 \\
\hline $4 \cdot 10^{4}$ & 4203 & 4235 & 0.9929 & 1.0005 \\
\hline $5 \cdot 10^{4}$ & 5133 & 5167 & 0.9935 & 1.0001 \\
\hline $6 \cdot 10^{4}$ & 6057 & 6077 & 0.9958 & 0.9990 \\
\hline $7 \cdot 10^{4}$ & 6935 & 6971 & 0.9928 & 0.9980 \\
\hline $8 \cdot 10^{4}$ & 7837 & 7867 & 0.9950 & 0.9988 \\
\hline $9 \cdot 10^{4}$ & 8713 & 8729 & 0.9949 & 0.9968 \\
\hline $10^{5}$ & 9592 & 9617 & 0.9961 & 0.9987 \\
\hline $2 \cdot 10^{5}$ & 17984 & 18013 & 0.9971 & 0.9986 \\
\hline $3 \cdot 10^{5}$ & 25997 & 26033 & 0.9965 & 0.9979 \\
\hline $4 \cdot 10^{5}$ & 33860 & 33919 & 0.9981 & 0.9999 \\
\hline $5 \cdot 10^{5}$ & 41538 & 41613 & 0.9983 & 1.0001 \\
\hline $6 \cdot 10^{5}$ & 49098 & 49151 & 0.9985 & 0.9995 \\
\hline $7 \cdot 10^{5}$ & 56543 & 56589 & 0.9982 & 0.9990 \\
\hline $8 \cdot 10^{5}$ & 63951 & 64071 & 0.9986 & 1.0005 \\
\hline $9 \cdot 10^{5}$ & 71274 & 71379 & 0.9988 & 1.0002 \\
\hline $10^{6}$ & 78498 & 78645 & 0.9983 & 1.0002 \\
\hline $2 \cdot 10^{6}$ & 148933 & 149033 & 0.9992 & 0.9998 \\
\hline
\end{tabular}


reason why the first crossing should occur at anywhere near as large an $n$ as Skewes' fantastic $10^{10^{10^{34}}},[18]$.

Applied Mathematics Laboratory, David Taylor Model Basin, Washington 7, District of Columbia

1. P. Chebyshev, "Sur une transformation de séries numériques," Oeuvres, v. 2, 1907, p. 707 .

2. P. Chebyshev, "Lettre de M. le professeur Tchébychev à M. Fuss, sur un nouveau théorème relatif aux nombres premiers dans les formes $4 n+1$ et $4 n+3$," Oeuvres, v. 1,1899 , p. $697-698$.

3. E. Phragmén, "Sur le logarithme intégral et la fonction $f(x)$ de Riemann," Öfversigt af Kongl. Vetenskaps, Akademiens Förhandligar, Stockholm, v. 48, 1891-1892, p. 559-616. 550 .

4. E. Landau, "Utber einen Satz von Tschebyschef," Math. Annalen, v. 61, 1905, p. 527-

5. E. Landau, Handbuch der Lehre von der Verteilung der Primzahlen, v. 2, Chelsea, 1953, p. 701-704.

6. G. H. Hardy \& J. E. Littlewood, "Contributions to the theory of the Riemann zeta function and the theory of the distribution of primes," Acta Math., v. 14, 1918, p. 127.

7. H. Bohr \& H. CramÉr, "Die Neuere Entwicklung der Analytischen Zahlentheorie," in Harald Bohr, Collected Mathematical Works, v. 3, Copenhagen, 1952, p. 804.

8. Daniel Shanks, "On the distribution of prime numbers in arithmetic progressions," Abstract, Goucher Meeting, May 2, 1959 of M. A. A.

9. H. F. SCHERK, "Bemerkungen über die Bildung der Primzahlen aus einander," Crelle's Journal, v. 10, 1833, p. 201-208. This table is more inaccurate than accurate.

10. J. W. L. GLAisher, "Separate enumeration of primes of the form $4 n+1$ and the form $4 n+3$, , Proc. Roy. Soc., v. 29, 1879, p. 192-197.

11. A. J. C. Cunningham, "Number of primes of given linear forms," Proc. London Math. Soc., v. 10, series 2, 1911, p. 249-253.

12. H. TiETze, "Einige Tabellen zur Verteilung der Primzahlen auf Untergruppen der Gruppe der teilerfremden Restklassen nach gegebenem Modul," Abhand. der Bayer. Akad. der Wiss., v. 55, new series, 1944.

13. JOHN LEECH, "Note on the distribution of prime numbers," Journal London Math. Soc., v. 32,1957, p. $56-58$.

14. G. H. HARDY \& Marcel Riesz, The General Theory of Dirichlet's Series, Cambridge, 1952 , p. 3.

15. Ramanujan, in a letter to Hardy, stated that these three classes were "equal". See S. Ramanujan, Collected Papers, Cambridge, 1927, p. 351.

16. Columns 1,2, and 4 of Table 7 agree with $\mathrm{H}$. TiETzE, Gelöste und Ungelöste Mathematische Probleme aus Alter und Neuer Zeit, v. 1, Munich, 1949, p. 25-26.

17. Ernst Trost, Primzahlen, Basel, 1953, p. 65-66.

18. S. SKEWES, "On the difference $\pi(x)-l i(x),(I)$," Journal London Math. Soc., v. 8, 1933, p. 278. 